\title{
Predictive Timing for Rhythmic Motor Actions in Schizophrenia
}

\author{
Yvonne Delevoye-Turrell*, Hélène Wilquin ${ }^{\dagger}$, and Mariama Dione *
}

Time is a common word, used frequently in our everyday language. It appears in many expressions: "I killed time playing cards," "Lets take time to talk," "I did two things at the same time." However, even if used by all, the word Time refers back to a complex phenomenon that is difficult - even impossible - to define. To this a priori simple question: "What is time?" the theologist Saint Augustin answered: "If no one asks, I well know; but if someone poses me the question, and I try to reveal my thoughts, I realise that I in fact do not know" (Les Confessions de Saint Augustin, Livre XXI, chapitre XIV, traduit de Moreau, 1864, édition 1942). Time has the ineffable character that reveals its existence only through a construct of the mind (Kant, 1845). Hence, Kants' understanding of Time infers two distinct phenomena: a physical absolute time and the psychological subjective time, the later being the only one sensitive to a distorted mind.

The physical time, encapsulated in the real number " $\mathrm{t}$," would correspond to that defined by Newton as Absolute Time: "Absolute time, without reference to anything external, flows uniformly" (1726 (1987), p. 408). Two concepts are here involved: absolute equality of time intervals ("uniform flow") and less obviously but equal essentially, absolute simultaneity, which is the more pervasive concept that underlies not only physics, but also the notion of past, present, and future. As such, the laws of physics impose to Time the idea of causality with the impossibility to act upon the past (i.e., to modulate events that have already taken place). The causality postulate may be one of the reasons why psychologists have revealed more interest in the study of Subjective time. The question being: "How does a subject experience the passage of Time? What is the experience of my own body as an actor in the past causing the occurrence of an event in the present time? Why can I, under certain situations, lose track of time with

\footnotetext{
* SCA Lab UmR CNRs 9193, University of Lille, rue du Barreau 59653 villeneuve d'Ascq.

$\dagger$ Aix-Marseille Université, LPCLS EA 3278, 13621, Aix en Provence, France.

(C) YVONNE DELEVOYE-TURRELL, HÉLÈNE WILQUIN, 2015 | DOI 10.1163/9789004230699_007
} This is an open access chapter distributed under the terms of the Creative Commons AttributionNoncommercial 3.0 Unported (CC-BY-NC 3.0) License, 
confusions between actions that I am performing and those that are to come in the future?"

It is the case that Time is in the hearts of our daily motor activities. Time helps structure our sensations and thoughts; Time is required for the production of coordinated motor sequences and voluntary interactive behaviours. Because movements involve changes in muscle length over time, motor control and timing are inextricably related. In the present chapter, we will consider the specific case of Motor Timing in Schizophrenia, a pathology affecting the mind and also the subjective experience of the passage of time. We will present a brief overview of the literature on subjective timing in adults suffering from chronic schizophrenia, starting from the patients' verbatim to the initial empirical data that has been reported. Then, we will present our own work on rhythmic production in schizophrenia to show the importance of developing new paradigms, based on more objective and non verbal paradigms. We will further try to show that these paradigms have the advantage of providing a better insight on the psychological mechanisms that may be at the origin of the distorted experiences of subjective time and agency in schizophrenia. We will finally conclude on the possible neurocognitive mechanisms that may underlie timing processes required for normalised motor control but that may also be the basis for the emergence of an anchored experience of the present time, psychological experience that is required for the construct of self as a unity of being.

\section{Subjective Time in Schizophrenia}

The challenge in psychology has been to study the sensation of time, a sensation that has no specific sensory system or organ - contrary to sight, hearing, taste, smell, and touch. A multitude of experimental paradigms have thus been proposed to study temporal processing in mind for: (1) the experience of being through time, (2) perceptual timing, and (3) motor timing. Substantial reviews have been published throughout the years about these diverse approaches (e.g., Bindra and Waksberg, 1956; Grondin, 2010; Repp, 2005). In this section, we will discuss a series of studies that reported the presence of time abnormalities in schizophrenia, with both explicit and implicit measures of temporal processing in the experiential and the sensory domains. But before, we first briefly describe the clinical aspects of the pathology.

\subsection{Schizophrenia: The Pathology}

Schizophrenia is a chronic, severe, and disabling mental disorder that affects approximately $1 \%$ of the population at some point in their lifetime. The 
disorder often develops earlier in men, usually in the late teens or early twenties, while women typically develop the disorder in their twenties and early thirties. People with schizophrenia often experience positive symptoms, e.g., delusions (believing that other people are reading their minds, controlling their thoughts or plotting to harm them), hallucinations (usually hearing voices not heard by others), incoherence, and physical agitation. Patients are also often identified with significant negative symptoms, e.g., disturbed interpersonal relationships, fearfulness, and social withdrawn. In later years, schizophrenia has been considered to be associated systematically to a disorganisation aspect that would affect all aspects of cognitive interplay, with disorganised speech, thoughts, emotions, and motor behaviour. Treatment almost always includes medications, with newer drugs being more effective (especially for the positive symptoms) and associated to fewer side effects than older ones.

While there is evidence that genetic factors have a role in the development of the pathology, other unknown causes play a significant part as well, suggesting a multifactor disease (Bellak 1949; Harrow and Jobe, 2007; Tsuang et al., 2001). While some proposed causes have been proven false, such as bad parenting and poor will power, there are many theories that remain. One of the most famous and most debatable is the dopamine hypothesis. The proposed hypothesis states that the brain of schizophrenic patients produces more dopamine than normal brains. It is this increased dopamine that is believed to be responsible for the symptoms of the disease. However, there is much debate in the scientific community as to the exact mechanisms by which altered dopamine levels, especially in the prefrontal cortex, striatum, and limbic system, produce schizophrenia (for a review, see Howes and Kapur, 2009). Evidence has nevertheless been presented through the last decade indicating that brain structures directly linked to neural timing processes are also impaired in schizophrenia (Volz et al., 2001), with the cognitive dysmetria hypothesis being central to the pathology (Andreasen, 1999; Andreasen et al., 1998). To note the origin of the term "dysmetria," which comes from the Greek term "metron," which means, "to measure a (time) scale or distance."

\subsection{Experiential Distortions of Time: Studying Verbatim}

The idea that time distortions are associated to schizophrenia is far from being a new hypothesis. Indeed, in 1932, Aubrey Lewis did a very nice report on the verbatim of patients suffering from schizophrenia. In their reports, patients describe different time abnormalities, e.g., a feeling of intemporality, the sensation of having the power to influence forthcoming events, phenomenon of premonitions and of "déjà-vus." For example, a clear description of a patient revealed a sense of time interruption with the individual stating that "Somebody 
was playing with the clocks" (Lewis, 1932, p. 612). In this statement, the patient was in fact describing his feeling that time was passing extremely fast at certain moments, but was extremely slow at others with seconds seeming to last an eternity. Another patient reported: "he would shoot gun fires towards his watch in order to kill time, which he considered as his worst enemy" (Minkowski, 1933, p. 13). More recently, a French psychologist transcribed examples of time distortions in young patients with Ultra High Risk of developing schizophrenia and with first-episode psychosis ( $15^{-25}$ years of age) for her PhD work (Wilquin, 2010). In her work, she reported, for example, a young patient who said to have found himself in front of closed gates at his high school on three different consecutive days, in the middle of the night. In a surprising way, this patient evoked the fact that he was unable to read the time on his watch as if the hands had temporarily disappeared. Another patient described that time passed much faster now (i.e., since his first psychotic episode) than before; and when talking about motor actions, the patient would state: "I make a movement but I have the impression that my body stays put," "I walk but I feel as if my body remains immobile..." These two verbatim are striking revelations of the possibility that in schizophrenia, time distortions affect even the most basic brain mechanisms enabling the coordination of (i) those motor commands sent out to the limbs for movement execution with (ii) the sensory (reafferences) information coming back from the contracted muscles.

Overall, the verbatim of patients have been helpful in providing an insight in the nature of the time distortions experienced by patients suffering from schizophrenia. Starting in the 1950's, more empirical studies were conducted in patients in order to gain a better understanding of the neuropsychological mechanism that may cause the temporal processing dysfunctions.

\subsection{The Initial Empirical Methodologies}

Various experimental paradigms have been developed in the field of time psychology, using more or less strict psychophysical constraints and in different perceptual modalities. Four basic methods have been commonly adopted to investigate sensory timing capacities of interval-durations. In verbal estimation tasks, participants are required to verbally report the duration of a presented stimulus ("the sound lasted 2 seconds"). The interval production tasks require participants to produce a target duration (by pressing continuously a key press or by marking verbally the onset and the end of the duration; e.g., beep...beep) that has been presented by the experimenter using verbal temporal units (e.g., "Could you please produce an interval-duration of 2 seconds?"). Quite similarly, in reproduction tasks, participants are asked to reproduce a time interval that they were presented with, but the time interval is presented here by a 
continuous stimulus. Finally, many variants of a comparative method have been used. This approach, which resembles the closest to the classic psychophysical paradigms, consists in the sequential presentation of two intervals (a standard or reference and a test interval). Participants are asked to say whether the test interval is shorter or longer than the reference interval (forced choice response). All these paradigms have been adapted to the specificity of clinical populations, the auditory modality being the most commonly used with a limited number of trial repetitions in order to consider the fatigability parameter.

The Goldstone and Lhamon (1956) study is one of the first to have assessed through empirical measures the distorted perception of time intervals in schizophrenia. A sound was presented to the participants, who were required to indicate whether this auditory stimulus was longer or shorter than a second. Results from this method of time interval comparison, revealed that all participants had a net tendency to overestimate the duration of the interval. Nevertheless, the overestimation was significantly longer for the patients with schizophrenia compared to the controls. Patients were also characterised by more variability in their responses.

In the following section, we report complementary results confirming these findings in patients with schizophrenia using these various experimental paradigms to investigate more specifically the nature of time perception distortions.

\subsection{Time Perception in Schizophrenia}

Patients diagnosed with schizophrenia display timing deficits. The exact nature of these deficits, however, varies across studies (Brown et al., 2005; Clausen, 1950; Densen, 1977; Johnson and Petzel, 1971; Rammsayer, 1990; Tysk, 1983a,b, 1990; Volz et al., 2001; Wahl and Sieg, 1980).

A majority of reports have used verbal estimation tasks, most probably because of the simplicity of the approach (no equipment required). These studies have confirmed temporal disturbances in schizophrenia, with a systematic overestimation of duration (e.g., a 10-s stimulus elicits a verbal label larger than $10 \mathrm{~s}$ ) in the seconds range (Johnson and Petzel, 1971; Tysk, 1983a; Densen, 1977; Wahl and Sieg, 1980). For example, Densen (1977) compared the results obtained in three different groups (10 patients with schizophrenia; 10 patients suffering from mental disorder other than schizophrenia; 10 controls) and reported a general tendency towards overestimation for intervals contained within the range of 5 to $120 \mathrm{~s}$ in the schizophrenic group of patients only and especially, for those short intervals of $5 \mathrm{~s}$.

When using the producing and the reproducing paradigms, results described systematic underproductions of temporal durations ranging from several seconds to one minute in schizophrenia, i.e., participants responded before $10 \mathrm{~s}$ 
had elapsed when required to respond at $10 \mathrm{~s}$ (Clausen, 1950; Elvevåg et al., 2003; Tysk, 1990; Wahl and Sieg, 1980). The studies conducted by Tysk (1983b) are interesting as they tested large patient groups $\left(\mathrm{N}=5^{0}\right)$ and showed that the timing deficits were found in the different subtypes of schizophrenia (following the DSM III classification) with an absence of symptom effects when contrasting positive and negative aspects (Tysk, 1990). Greater differences in time estimation of visual vs. auditory signals have also been reported in patients when compared to controls (Penney et al., 2000).

In the past twenty years, more contemporary paradigms, i.e., temporal discrimination tasks, have dominated research on time perception in schizophrenia (Carroll et al., 2008, 2009a; Davalos et al., 2003; Elvevåg et al., 2003; Rammsayer, 1990). For example, Elvevåg et al. (2003) employed brief stimuli so that the use of certain strategies, such as counting, would be reduced or eliminated (short time scales $<2 \mathrm{~s}$ ). In their study, participants were asked to perform two different tasks. In the temporal generalization task, participants were initially presented with a standard duration tone (50oms) and during the test phase they were asked to give interval duration judgments with standards of various durations $\left(125,250,375,625,75^{\circ}\right.$, and $\left.875 \mathrm{~ms}\right)$. Results showed that patients made overall more mistakes but differences laid especially for those intervals greater than 50oms. These deficits could not be due to contrasting learning effects between patients and controls. A temporal bisection task was also used. Here, there were two standards, one long and one short, and participants were asked to judge whether test stimuli were more similar to the long or to the short standard. Once more, patients performed more mistakes but statistically reliable differences were found for those intervals greater than 6ooms only. In both tasks however, patients were characterized by flatter gradients than controls, indicating less accurate and especially overall more variable timing capacities. Using a similar comparative method, Wilquin et al. (2010) and Ameller et al. (2011) also reported preserved perceptual detection of irregularities in otherwise regular sequences for interval shorter than $500 \mathrm{~ms}$, even if once more patients revealed overall more errors in their decision making.

Considering all in all, patients with schizophrenia seem to experience time as lengthened relative to objective time, especially for inter-tone intervals greater than 6ooms, which is consistent with patients' subjective reports of an elongated experience of the passage of time in daily activities (Freedman, 1974). These findings have generally been interpreted as reflecting an increase in speed of a hypothetical "internal clock" (Johnson and Petzel, 1971; Tysk, 1990 - for theoretical basis to the question, see Gibbon and Church, 1984), an interpretation that has been assumed consistent with animal studies of the effects of dopamine agonists on timing (Rammsayer, 1990). We will come back 
to this concept of internal clock in Section 3 of the present chapter. However, while these results may reflect temporal deficits in schizophrenia, they may also be due to other (cognitive) dysfunctional processes. Specifically, global deficits in verbalisation, motivation, or even attention capacities may also affect performance levels in verbal estimation tasks (Brébion et al., 200o). Yet an additional possibility is that the overestimations and underproductions are a consequence of systematic memory distortions (Penney et al., 2000). Indeed, recent studies have observed significant correlations between the performance on time perception tasks and that found in neuropsychological outcomes typically affected in schizophrenia (e.g., Lee et al., 2009; Wilquin et al., 2010).

This limit of the verbal estimation paradigms, especially when working in clinical populations, has led to the use of non-verbal, non-decisional tasks in order to gain a more accurate (pure) assessment of timing deficits in schizophrenia.

In the following section, we report findings obtained for motor timing in adult patients suffering from schizophrenia in various motor sequential tasks in order to gain a better understanding of the nature of the timing processes that may be impaired. With this aim, we will now report those studies investigating timing deficits using synchronization-continuation finger-tapping tasks, a paradigm that does not require verbal or explicit report. Here, we have taken the position that motor timing and time perception rely on overlapping (and maybe identical) brain circuitries (Burr et al., 2007; Ivry and Hazeltine, 1995; see Buonomano and Laje, 2010 for a counter discussion).

\section{Motor Timing to Assess Subjective Time in Schizophrenia}

It is the case that a number of studies of human timing have indicated that accurate estimation of intervals in the order of several seconds requires sustained focusing of attention and/or memory (Fortin et al., 1993; Fortin, 1999; Zakay and Block, 1996). Furthermore, strategies such as counting can be recruited in making such judgments of interval durations. The involvement of attentional and strategic processes in these perceptual tasks is noteworthy given the well-documented reports in schizophrenia of systematic attentional impairments (e.g., Cornblatt and Keilp, 1994; Delevoye-Turrell et al., 2006; Nuechterlein and Dawson, 1984), and working memory deficits (e.g., Fleming et al., 1997; Stone et al., 1998). Hence, it is important to consider whether the systematic findings of perturbed timing processes in schizophrenia - reviewed above - reflect general impairments in cognitive processes rather than a specific difficulty with temporal processing (Rammsayer, 1999). 
With this in mind, we have started to develop an original approach consisting in adapting the synchronization-continuation finger-tapping paradigm (Stevens, 1986) to pathological populations. Such a task requires participants to first tap in time with computer-generated tones separated by a fixed intertone interval (tone-paced tapping). After a series of tone-paced responses, the tones are discontinued and participants are required to continue. Using measuring devices like button presses, force transducers, and tactile screens, we have investigated the capacity of individuals to tap in rhythm to a metronome. Without explicit instructions to focus attention on the temporal information, participants perform the task as a simple rhythmic motor task. Importantly, the difficulty of the task can be manipulated experimentally by varying the complexity or length of the tapping sequence to produce; the rhythmic pattern can be maintained but task difficulty modulated by changing the spatial relationship between successive taps; and the pleasure experienced by the participants can be also modulated by changing the types of sounds the participants are required to synchronise to (tap to the sound of marching feet or to your favourite pop tune vs. tap to the metronome beat) - a nice approach to limit boredom.

We have used adapted synchronization-continuation tasks in order to work with different age groups of participants and with individuals suffering from different types of mental illnesses, with limited influences of associated cognitive abnormalities in memory, IQ or attention capacities. Thus, in the following sections, we report some of our recent findings obtained with these original motor timing tasks in patients suffering from schizophrenia (the "Touch 2 Know" tablet application).

Before, however, we wanted to emphasize in a first section the fact that accurate timing is crucial for motor control, especially for the fluency of motor sequencing. More precisely, we report data obtained in patients performing different types of sequential motor actions that require the precise timing of each sub-movement of the sequence in order to achieve motor goals.

\subsection{Sequential Motor Control in Schizophrenia}

Motor control, from catching a ball to playing a musical instrument or dancing a waltz, requires the production of complex spatiotemporal patterns of muscle activity. As such, most motor tasks, including speech production, require the carefully orchestrated movement times in the order of tens of milliseconds to a few seconds. In a task where a single effector is used to perform a sequence, each successive sub-movement is dependent on completion of the preceding one. It takes an appreciable amount of time for peripheral sensory information to reach the central nervous system, and in the presence of such delays, it is generally suggested that motor sequences may be timed in 
two fundamentally different ways depending on whether or not they require sensory information. First, sequencing may be based on concurrent processing of sensory information (Macefield and Johansson, 1994). For such triggered sequences, each element in the sequence will be initiated only after the arrival of the sensory feedback signalling the end of the preceding element. An alternative approach involves preplanning the entire sequence before its initiation as a whole (Billon et al., 1996). In this case, the sequence is represented as a single entity and performed without reference to feedback from external events in a pre-planned fashion. The correct preparation and execution of such planned sequences rely on well-defined predictive processes developed through previous experiences and depend on the brain's ability to organize through time and space information from multiple sources.

In the psychiatric literature of schizophrenia, many reaction time studies reported general slowness in schizophrenia (Cohen and Servan-Schreiber, 1992; Sereno and Holzman, 1996; for a review, see Nuechterlein, 1977). However, it is the case that the selected tasks often required sequencing over time, for example, detect a white dot and then detect a blue dot and then press the switch as soon as the blue dot disappears (Zahn et al., 1998). Hence, it is possible that slowness is not characteristic of patients with schizophrenia but arises in these patients because timed sequencing is necessary.

In a series of sequential motor tasks, we used a simple load cell to assess whether the motor slowness in schizophrenia was directly related to the need to sequence a series of actions through time (Delevoye-Turrell et al., 2003). Furthermore, through the implementation of different types of action sequences (triggered vs. planned), we were able to dissociate between lower and higher timing mechanisms for the deficit. Overall, results demonstrated that schizophrenia is associated to higher timing mechanisms only. Indeed, for triggered sequences, the participants' task was to let an object slip between fingertips and to augment grip force to arrest object fall. Results showed that the group mean time delays between two successive motor elements were similar in patients and controls, supporting the hypothesis of preserved lower order (subcortical) integrative mechanisms in schizophrenia (Delevoye-Turrell et al., 2007). For planned sequences, participants were required to press a series of flat button that were displayed on a table top, one after the other, as fast as possible at a stop signal (i.e., following a regular and fast rhythmic pattern). For the controls, the time delay between successive elements was short (40ms) affording a fluent and fast execution of the rhythmic pattern, suggesting that the timing of the motor elements was pre-programmed without the need of external sensory information (Billon et al., 1996). A different pattern of results was obtained in 
the patients with performances that were characterized by inter-movement delays that were: (a) longer for planned than for triggered sequences, (b) abnormally long compared with the controls, and (c) increased with sequence timing complexity. Overall, these data support the possibility that schizophrenia is characterized by a lack of fluency in the execution of planned sequences, which would be related to a higher order deficit in the temporal sequencing of multiple elements for motor fluency. This high order timing deficit may be a plausible explanation for the impaired ability to detect a mismatch between a selfgenerated movement and its consequences, but not impaired in their ability to automatically compensate for low level gain change in a continuous circledrawing task (Knoblich et al., 2004), which may require two complementary timing mechanisms (Robertson et al., 1999; Zelaznik et al., 2000).

After presenting findings from sequential motor experiments in patients with schizophrenia, we continue in the next section, by reporting experimental studies tailor-made to assess motor timing distortions in schizophrenia during the planning and execution of rhythmic motor sequences.

\subsection{Motor Timing in Schizophrenia}

Elveväg and collaborators (2004) were among the first that tested patients with schizophrenia in a synchronization-continuation finger-tapping task to an auditory beat, with a metronome at 30 or 60 beats per minute (bpm). After a 5 -minute period, the metronome was then switched off and participants were to continue as before for $5 \mathrm{~min}$, but now were required to generate the rhythm internally. Results revealed that all participants were rather accurate during the synchronisation task (unpublished data) but when asked to tap without the metronome, controls were better able to generate a constant tempo with minimal fluctuations than were patients. Interestingly, in the 30 -bpm task patients disproportionately speeded up their responses over time. Note that the patients were reported being better when asked to perform faster rhythmic patterns (6o-bpm) than slower ones (3o-bpm) - we will come back to this point in the last section of this chapter.

Carroll et al. (2009b) used a similar synchronization-continuation fingertapping task with an auditory tone paced at 50oms inter-tone intervals $($ ITI $=500 m s)$. Thirty-two participants with schizophrenia displayed significantly faster tapping rates, with significantly shorter ITIs, for both tone- and self-paced portions of the task compared to the non-psychiatric group. They also displayed greater tapping variability during both tone- and self-paced portions of the task. In a second analysis, the Wing-Kristofferson model (Wing and Kristofferson, 1973) was applied to the self-paced data to determine whether increased tapping variance in schizophrenia should be attributed to clock or 
motor implementation factors. Consistent with the temporal representation demands of the self-paced task, clock variability was significantly higher in the schizophrenia group. Estimates of motor implementation variance did not differ between individuals with schizophrenia and controls. The partitioning of variance into clock and motor factors hence suggested that group differences in tapping variability were largely due to decreased temporal precision of the internal clock, in schizophrenia.

Still using a synchronization-continuation finger-tapping task, we wanted to go further by assessing the effects of task difficulty and attention demands for motor timing during motor sequencing. For this reason, we manipulated the complexity of the rhythm and/or the complexity of the motor task to perform motor sequences in synchrony with an external metronome (Wilquin et al., 2010). The participants' task was to squeeze a force transducer in its centre in beat with a metronome for a 12-s period, and then to continue producing the same rhythm in a continuation phase for another 12-s period (Figure 6.1left). In order to manipulate task difficulty without modifying rhythm complexity, two pitch tones were used. Participants were required to produce strong squeeze forces for high pitch $(16 \mathrm{~N})$ and small squeeze forces for low pitch $(8 \mathrm{~N})$. These pitches were presented alternating providing the participants with the possibility to predict the rhythmic sequence to perform well before the start of each trial. In a second condition, we manipulated rhythm complexity without modifying task difficulty. Here, IT Is of 300 and $700 \mathrm{~ms}$ were
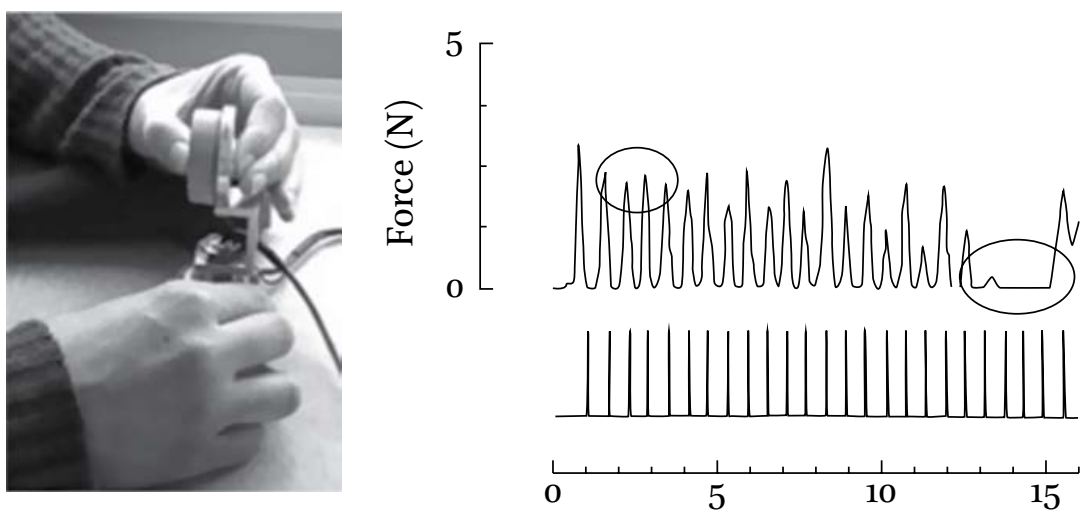

FIGURE 6.1 Picture of the experimental apparatus used to measure squeeze forces produced on the load cell with the participants' dominant hand (example of a left-handed patient with schizophrenia) in function of a metronome (left). Example of an equivalent rhythm for which subjects were required to alternate force levels in function of high and low pitch tones as a function of time (in seconds). Note the difficulty in force alternation but also of the presence of an attentional lapse twelve seconds after the start of the trial (right). 
alternated with a single pitch tone for which participants were to use a $12 \mathrm{~N}$ squeeze force. Results revealed that in the simplest of conditions (equivalent force/equivalent time), patients and controls performed very few production errors and were highly accurate with mean errors smaller than 1oms, which is similar to that classically reported in the literature (for review, see Aschersleben, 2002). With motor task complexity (alternated force/equivalent time), patients were in difficulty and were characterised by more rejected trials. A more specific analysis of these errors confirmed that patients were characterised by more frequent arrests in rhythm execution (Figure 6.1-right), attentional lapses that are consistent with the clinical lapses frequently noted during semistructured interviews of patients with schizophrenia (Ameller et al., 2011). Nevertheless, for those trials (alternated force/equivalent time) performed correctly and without lapses throughout the 24-s period, timing errors were similar in controls and patients, suggesting a problem of attention for action execution (Delevoye-Turrell et al., 2006) rather than a timing problem per se.

Interestingly, in both conditions for which the rhythm complexity was manipulated (equivalent force/alternated time; alternated force/alternated time), patients revealed on those trials correctly performed, significant timing errors both during the synchronisation and the continuation phases (Figure 6.2). These errors were not correlated with the treatment dosages or age; they were slightly correlated to the attention capacities as evaluated by the $\mathrm{D} 2$ test for attention (i.e., the participants are required to cross out as many $d$ 'symbols that are mixed up with $p, d$, and $d$ ' symbols within a timed period of 1 minute - see Brickenkamp, 1962). Following the theories developed for dynamical systems, sensorimotor synchronisation for equivalent time intervals would need the same timing mechanisms than that used for continuous movements (Zelaznik et al., 2005). It would require minimal attention resources because it would be highly automatic (Pressing, 1999) emerging from the inherent timing properties of the neural circuits processing motor planning and execution. For this low-level processing of motor timing, patients with schizophrenia would not be perturbed and thus, they show similar timing capacities than controls. For alternated interval timing, a more explicit control would be required in order to process each interval as a discrete time series (Pressing, 1998). Here, participants would be required to maintain cognitive representations of time interval durations, and order thanks to the existence of a kind of internal clock. Predictive timing would thus be involved, an anticipatory cognitive mechanism that would be impaired in schizophrenia. Because of the need of significant cognitive resources, alternated interval-timing tasks are more difficult to execute even for healthy controls than equivalent intervaltiming tasks (Delignières et al., 2004; Zelaznik et al., 2002). 

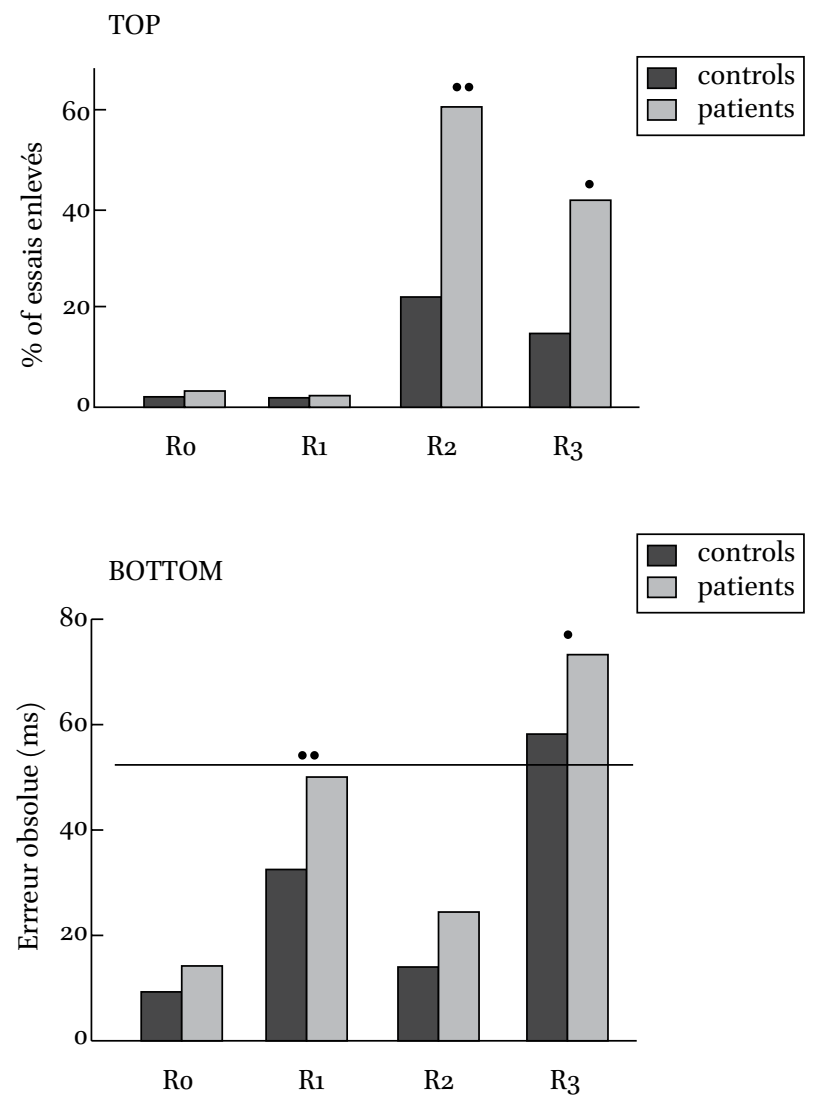

FIGURE 6.2 Number of bad trials measured in healthy controls (black) and patients with schizophrenia (grey) in a synchronisation task requiring subjects to perform a series of squeezes on a load cell synchronisation with equivalent time intervals (Ro; R2) and alternated time intervals $\left(R_{1} ; R_{3}\right)$ with equivalent force levels $\left(R o ; R_{1}\right)$ or alternated force levels $\left(R_{2} ; R_{3}\right)$. Results showed that for both controls and patients, it was difficult to alternate force levels and maintain the timing motor task but patients failed more often than the controls (top). When assessing absolute timing errors on those trials performed correctly (bottom), results demonstrated that patients made significantly more timing errors than controls in those conditions for which alternated time intervals were to be performed. Overall, these results suggest a specific difficulty in predictive timing in schizophrenia.

In the following section, we present two studies that were conducted specifically to test the hypothesis that the abnormal performance patterns reported both for time perception and motor timing in individuals with schizophrenia may be associated to a distorted internal clock for temporal processes. The inconclusive findings will lead us to propose in a fourth and final section a 
novel spatio-temporal paradigm that has given us the means to reveal in schizophrenia a specific problem of the anticipatory process that is necessary for predictive timing.

\section{4}

\section{A Preserved Internal Clock in Schizophrenia}

The overestimation and underproduction results described above for both time perception (Section 2.4) and motor timing (Section 3.2) suggest that the representation of time is severely distorted in schizophrenia. In the following section, we report two fundamentally different protocols that were developed in our laboratory to test the degree of normality of the pulse rate of a possible central oscillator (internal clock) for explicit and linear metric of time in schizophrenia.

\subsection{Time Decay of Body Schema}

Numerous contributions, mostly clinical reports and phenomenological considerations, have been published on body image pathology in schizophrenia (for reviews, see e.g., Fisher, 1986; Priebe and Röhricht, 2001). Schizophrenia patients are reported to suffer from symptoms of disembodiment such as not feeling at home in one's body anymore, feeling disintegrated, or feeling as if the body is torn apart, suggesting a problem in integrating different sources of information in a unified body representation. Explanations vary and range from neurological to psychological perspectives (Cumming, 1988). In the following study, we wanted to quantify the degree of abnormalities of body image in schizophrenia but especially assess its decay over time in order to get an insight on the phenomenology of the passage of time and its role in a possible distorted body image in schizophrenia.

For this purpose, we adapted the head "return" paradigm, with which one can dissociate the perceived orientation of a body part from its actual position without manipulating proprioceptive information. The so-called head "return" phenomenon was described by Gurfinkel et al. (1989) as the perception of a slow head movement toward the mid-sagittal plane, i.e., its neutral position, while the head is kept turned about 10 minutes with eyes closed. The head "return" phenomenon has since been studied by many researchers and it has been proposed that the illusory displacement of the body segment is due to the decay over time of the body image. In the present case, we studied an arm "return" phenomenon in patients with chronic schizophrenia to assess whether the decay over time would be faster than in healthy controls, under the effects of a faster ticking internal clock. 
Twenty-nine patients with schizophrenia (10 females, mean age $=36$, SD $=10.7)$ and thirty healthy controls (12 females, mean age $=34.5, \mathrm{SD}=14.4$ ) were tested in a simple session (unpublished data from Gorog et al., 2005). All patients fulfilled the DSM-IV criteria for schizophrenia and three were drug-free. Participants were invited to seat in front of a table, facing a curtain that prevented them to see more than $30 \mathrm{~cm}$ in front of them. They were asked to passively let the experimenter put their (right) target hand behind the curtain, flat on the table, rotated with a $30^{\circ}$ angle from the body's straight ahead axis. During the entire session, participants were required to maintain this body position, with shoulders and back straight, with minimal muscle contractions of upper limbs and no visual feedback of the hands.

At the experimenter's signal, the participants were required to move their (left) pointing hand in a symmetrical position from the target hand, relative to the body sagittal axis, and place their palm flat on the table. When set, the positions of both hands were measured on a bidirectional scale $(\mathrm{X}, \mathrm{Y})$ in centimetres $(\mathrm{cm})$ with point zero being aligned with the participants' body sagittal axis. The experimenter was careful not to touch the participants' hand. With the measurements completed, the participants were required to bring their pointing hand back to the resting position, which was tactilely detectable with the help of a small rough surface that was placed on the table close to the participants' midline. They were asked to repeat this exact same procedure at five critical moments: $0,1,3,5$, and 8 minutes at the experimenter's signal.

Results revealed a significant Group effect for the error of initial hand position at To $[F(1,43)=10.704, p<0.01]$. Twenty-seven patients out of 29 were characterised with a greater error $(\mathrm{ME}=12.1, \mathrm{SD}=10.6 \mathrm{~cm})$ than that obtained in the control group $(\mathrm{ME}=0.8, \mathrm{SD}=5.9 \mathrm{~cm})$. Interestingly, errors at To for the patients were positively correlated with the negative score of the PANSS ( $R=0.43$, $\mathrm{p}<0.02$ ), suggesting greater initial distortions of body image for patients with more severe negative symptoms (even when controlling for treatment dosage). More importantly for the scope of the present chapter, the effects of Time on the decay of body image was highly significant $[\mathrm{F}(1,43)=15.05, \mathrm{p}<0.001]$, with similar slopes for the linear regressions in both groups $[F(1,43)=0.490$, $\mathrm{p}=0.481]$. This preserved "return" phenomenon in schizophrenia is illustrated in Figure 6.3.

Overall, these findings indicate that over the 8-minute period the effects of time gave the participants' the illusion of the hand progressively being closer to the body's sagittal axis. But importantly, the ticking clock decay was similar in the healthy controls and in the patients with schizophrenia. 


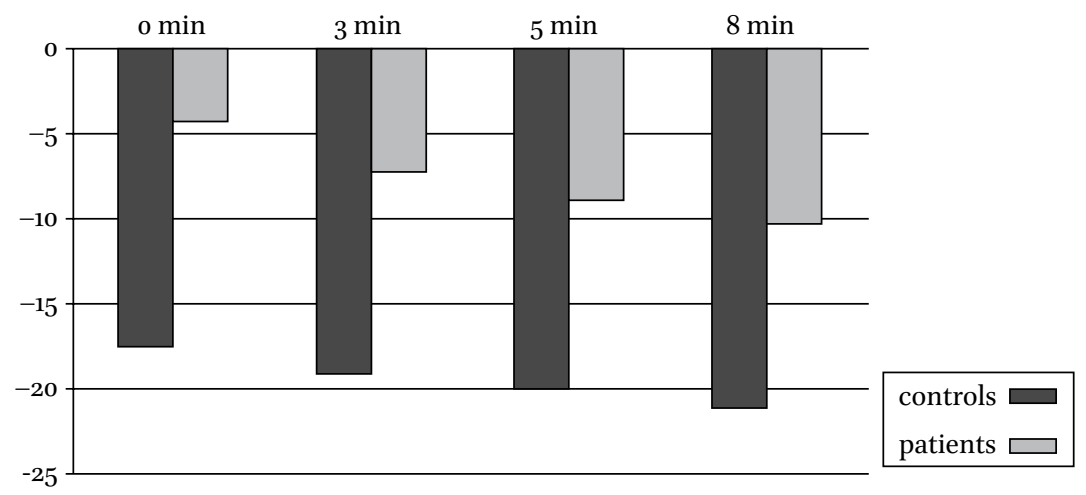

FIGURE 6.3 Graphical representation of the errors in body schema observed in patients with schizophrenia (red) and controls (blue) during the arm "return" paradigm at four critical moments: o, 3, 5, 8 minutes from the start of the experimental session. Note that reproduction errors of hand symmetrical position compared to the subjects' body sagittal axis increases at a similar rate for both subject groups suggesting a normalised ticking clock decay in schizophrenia.

\subsection{Ticking Frequency of the Internal Clock}

Within the framework provided by pacemaker-accumulator models of interval timing (Gibbon et al., 1984), a faster internal clock than normal clock speed would result in a shift in an estimate of duration only when the experience of time on the test trial is measured with a faster clock than that used to measure the reference duration (Meck, 1996). Given the same contingencies in a verbal estimation task, the findings for patients with schizophrenia might be interpreted as due to differential clock speeds for reference and comparison durations, suggesting a less stable clock ticking frequency. To test this hypothesis directly, the spontaneous tapping task has been proposed for which participants are required to produce a regular sequence of finger taps (Vanneste et al., 2001). This measure has the advantage of requiring no subjective or explicit decision-making as well as no need for explicit memory capacities.

In a recent study, we measured spontaneous tapping tempo and used a touch screen in order to measure Inter Response Interval (IRI), i.e., the dependent variable traditionally measured in finger tapping studies. However, thanks to technological advances, we were able to decompose each IRI as the sum of two new variables that helped us dissociate timing distortions from motor control dysfunctions (Delevoye-Turrell et al., 2012). First, Contact Duration (CD) was defined as the time interval during which the finger is in contact with the screen. This indicator is associated to the time needed by the sensorimotor system to detect that the finger has made contact (finger down) and that the 
next element can thus be initiated (finger up). Hence, contact duration is an indicator of the brain capacity to quickly integrate incoming sensorimotor (haptic) afferences with outgoing motor efferences. The second parameter is the Flight Time (FT), i.e., the time during which the finger is moving from one target to the next. This parameter is planned in a predictive manner on the basis of an open loop that cycles at the ticking frequency of the internal clock (finger down...finger down...). As such, it does not depend on sensorimotor integration but requires clock-based mechanisms and may, thus, reveal the pure ticking frequency of a possible timer.

Twenty-four patients and 22 controls participated in the study. Participants were seated comfortably on a chair in front of a touch screen (Elo Touch, $23 \mathrm{~cm}$ $36 \mathrm{~cm}, 30 \mathrm{~cm}$ ), which was placed on a narrow support at knee-height. Each trial started with the presentation of a visual image that was made of 6 black circles (Figure 6.4-left). The participants' task was to point each circle one after the other, clockwise, starting from the bottom-right circle. Participants were asked to use their preferred hand and to tap as regularly as possible, at their preferred rate. No sound was ever heard. A trial was ended after the production of 30 taps, and a total of 12 trials were recorded.

Results revealed that patients with schizophrenia were able to produce regular sequences of spontaneous taps but at a slower rate than that measured in age-matched controls (IRI of 610 vs. $487 \mathrm{~ms}$ for patients and controls, respectively). These results are consistent with previous studies reporting (1) IRI of around 500ms in healthy adults (McAuley et al., 2006) and (2) general movement slowness in patients with schizophrenia (Morrens et al., 2007). Interestingly, tempo slowness was positively correlated to the slowdown of cognitive speed in patients, which suggested a central origin for the deficit (Figure 6.4-right).

However, through the use of a touch screen with an original time performance subdivision, our analyses revealed that an overall mean FT of $349 \mathrm{~ms}$ was displayed across experimental groups $(328$ and $369 m s$ for controls and chronic patients, respectively). Coherent with an absence of group effect, our results argue in favour of a normalised ticking frequency of the internal clock in chronic and stabilised patients with schizophrenia, with furthermore similar timing stability (FT variability) in both patients and healthy individuals. These results resemble those reported in the early 8os in studies investigating spontaneous eye-blink rates, with normalised spontaneous rates in stabilised patients under neuroleptic treatment (Karson, 1983). Our chronic patients were characterised nevertheless with significantly longer CDs than controls (see Table 6.1), which argues in favour of a specific problem in the fluent production of the finger down/up rhythmic movement in schizophrenia. 


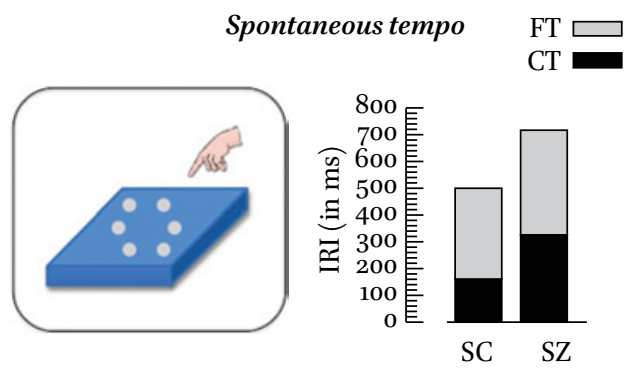

FIGURE 6.4 Illustration of the experimental setup used to measure spontaneous tempo in controls and patients with schizophrenia (left). Results showed that tempo was slower in patients but this difference was solely due to a significant increase in contact duration $(C D)$ of finger on the screen. Flight time (FT) was similar across groups arguing in favour of a normalised ticking frequency of the internal clock in schizophrenia (right).

In conclusion, it is possible that the general psychomotor slowness and especially distorted temporal processes are not related to frequency abnormalities in a central oscillator that would play the role of a possible internal clock. We favour here the hypothesis of a specific deficit in the rhythmic timing of action sequences, i.e., a deficit in the sensorimotor integration of predicted incoming afferences with outgoing efferent commands.

\section{$5 \quad$ Tempo Variations to Reveal Specific Timing Processes}

In the field of visual perception, authors have suggested difficulties in discriminating simultaneity during the presentation of visual stimuli in schizophrenia, findings that may suggest a specific impairment in time event coding (Foucher et al., 2008; Giersch et al., 2009; Lalanne et al., 2010; see Chapter 5 of this book). With a difficulty to distinguish physical past and present events, the coding of the passage of time could be impaired with direct consequences on the capacity to correctly time and integrate sequences of multiple events. In the following section, we present the idea that the time-dependent properties of a task may induce two different timing processes for perceptual and motor timing. A new protocol in which the tempo is manipulated will be proposed to assess whether synchrony disturbances may be present only in certain time spectrum, suggesting a specific deficit in predictive timing in schizophrenia. 
TABLE 6.1 Mean values (standard deviation) obtained in healthy controls and patients with schizophrenia for rhythm production corresponding to spontaneous tempo, with the subdivision of inter-response-intervals (IRI) in Contact Durations (cD in ms) and Flight Times (FT in $m s)$. Note that patients are as regular than controls in their rhythmic production.

\begin{tabular}{lllll}
\hline & $\begin{array}{l}\text { Spontaneous } \\
\text { Tempo (IRI) }\end{array}$ & $\begin{array}{l}\text { Regularity } \\
\text { ratio }\end{array}$ & $\begin{array}{l}\text { Sensori- } \\
\text { motor integration CD }\end{array}$ & Internal clock FT \\
\hline $\begin{array}{l}\text { Controls } \\
(\mathrm{N}=22)\end{array}$ & $487(107)$ & $1.004(.007)$ & $161(74)$ & $328(78)$ \\
$\begin{array}{l}\text { Patients } \\
(\mathrm{N}=30)\end{array}$ & $610(155)$ & $1.010(.010)$ & $246(133)$ & $369(115)$ \\
\hline
\end{tabular}

\subsection{Two different Timing Mechanisms for Interval Timing}

Analyses of the neural basis of timing have generally focused on two general computational strategies: mechanisms based on neural clocks and mechanisms that emerge from the dynamics of neural networks. In general, these models must accomplish some variant of the same computational task by recoding the temporal information present in the (sensory) input into a spatial (motor) code. Hence, different cells must respond selectively to temporal features of the stimulus.

When considering the mechanisms of timing it is perhaps most intuitive to think in terms of clocks or interval timers. The basic computational unit of clock theories involves an oscillator and a counter (Creelman, 1962; Treisman, 1963). Conceptually, in this top-down approach, the oscillator beats at some constant frequency and each beat is then counted by some sort of neural integrator. These clock-like mechanisms could be involved in timing on the scale of seconds and minutes (Meck, 1996; Matell and Meck, 200o) but would clearly be, at the neurophysiological level, in difficulty for shorter intervals. We consider an alternative bottom-up approach to be interesting when considering short time intervals $(<1 \mathrm{~s})$. Here, starting from biologically realistic assumptions, authors have asked the extent to which temporal processing can be found as an emergent property. Then, it has been shown that cortical networks are inherently able to process temporal information because information about the recent input history is inherently captured by time-dependent changes in the state of the network (Buonomano and Merzenich, 1995; Buonomano, 2000; Maass et al., 2002). Given the high dimensionality and abundance of time-dependent properties of cortical networks, this type of model could provide a realistic means to decode complex 
temporal and spatial-temporal patterns of sensory information (Buonomano and Laje, 2010). As such, there would be no need of a centralized clock for temporal processing on the scale of tens to hundreds of milliseconds, but interval timing for fast tempi and complex temporal patterns would be accomplished through non-specialised network dynamics (Buonomano, 2000; Maass et al., 2002).

Ivry et al. (1988) provided one of the first neurophysiological evidence that central (clock) timing and emergent timing processes are independent. Patients with a lesion only in the medial region of a cerebellar hemisphere exhibited increases in emergent timing variance for the ipsilesional hand, compared to the contralesional hand, whereas those patients with localized lateral damage to a cerebellar hemisphere exhibited increases in central clocklike variability, only for the ipsilesional hand compared to the contralesional hand. Moreover, patients with cerebellar lesions also were impaired on a variety of sensory interval-timing processing tasks, suggesting that this structure operates as an internal timing system that is exploited for both motor and non motor tasks, which require the precise representation of temporal duration (reviewed in Ivry, 1997).

In an elegant and systematic analysis of timing aspects for motor control, Zelaznik and collaborators explored the idea that the timing of movement initiation processes involves an explicit representation of time, whereas movements that are continuous may not (Zelaznik et al., 2002; Robertson et al., 1999). They hypothesized furthermore that once initiated, continuous movements no longer involved explicit temporal control. Rather, the temporal regularities would be emergent, reflecting the implicit operation of a different control mode. For this, the authors used three different tasks in a classic synchronisation-continuation paradigm. Two tasks were used in the discrete condition: a finger-tapping movement and an intermittent circle drawing task, for which participants were required to insert a pause between each drawing cycle. In the continuous condition, participants were asked to perform the circle-drawing task without intermittence. Results showed that temporal consistency on the tapping and intermittent circle drawing tasks was significantly correlated in all of the experimental situations, and performances on these tasks were also correlated with acuity on a duration discrimination task. In contrast, the correlations were either weaker or absent between these tasks and the continuous circle drawing task. While all of the tasks could be considered as "timing" tasks, one fundamental difference here between the tasks in the discrete condition, on the one hand, and the task used in the continuous condition, on the other hand, was the need to explicitly time the end and beginning of each sub-movement, in the discrete condition. 
In the above example, the two timing modes were revealed through the use of two different motor tasks. It is, thus, difficult to ensure that all other features of the motor planning and execution steps were identical and that the only differing parameter was the temporal aspect. In the following section, we present a unique synchronisation task that provided the means to investigate the accuracy and the stability of explicit and implicit timing mechanisms in both healthy controls and patients with schizophrenia.

\subsection{The Spatial-tapping Task and Schizophrenia}

When walking down the street, at a slow pace, it frequently happens that we control the end position of our foot. This may especially be the case in dirty streets to avoid dog-poo or mud holes. Now imagine walking to catch a bus that is on the verge of departing. Now, your steps will be executed at a higher tempo; you may not think of the cleanness of your shoes because your primary goal is to catch that bus. The Spatial Tapping task is based on this simple idea and requires participants to perform a synchronization task with a metronome that sets different tempo speeds. Contrary to all classic finger-tapping tasks, the participants will in addition need to move through space tapping in a counter clockwise order a series of 6 targets disposed around a circle (see Figure 6.4left). As such, there is both a timing aspect and a spatial dimension to the task. We predicted that at slow tempi, participants would explicitly control the sequential timing of the initiation of each finger-tap; at fast tempi, the objective would be to move the arm around the global circle, keeping as best possible the overall timing of the cycle dynamics. In this later case, the timing of each individual tap would thus become emergent, slave to the overall timing of the arm movement. To test our hypothesis, we performed analyses on the endpoint distributions both in time and space in order to confirm the presence of two different temporal processes in function of imposed tempo.

In the motor control literature, the analysis of endpoint distributions of hand pointing actions has revealed the existence of different planning strategies depending upon task demands (Gordon et al., 1994). Inspired by the findings of Gordon and collaborators, Desmurget et al. (1998) demonstrated that the dispersion properties of endpoint distributions of single pointing movements - from a starting point to a visual target - could be influenced through instructions. When no other instructions were given than executing a pointing movement towards a visual target, Desmurget et al. observed that the distribution of the endpoint errors around the target was roughly circular. However, with the specific instruction to 'follow a straight line path', the endpoint distributions of the pointing actions were elliptical in shape, with the major axis being systematically oriented along a virtual line joining the 
starting point and the final visual target. Similar results have been published by others, suggesting that endpoint ellipses emerge as oriented when the motor plan is guided by an internal representation of grouped targets (Vindras and Viviani, 1998).

Recently, we applied the analysis of endpoint ellipses (orientation and area) to the results obtained in the finger-tapping synchronisation task to reveal distinct control (executive) strategies in relation to two different temporal control processes for various imposed tempi. Eighteen controls and 18 patients with schizophrenia participated in a unique experimental session. After sitting down in a silent room for 5-min (brain-clearing phase for rhythmic cues), participants were invited to produce a spontaneous series of 60 touchdowns on a tactile screen. The explicit instruction was as follows: "Point each target, one after the other, at your preferred speed...one that seems the most comfortable to you. Try to be as regular as possible." Customised software (written in MATLAB programming language) calculated the participants' spontaneous tempi and then, the software proposed a series of 11 trials at various tempi at and around, the middle-spontaneous tempo. On average, the spontaneous tempo was similar in patients and controls and was close to $600 \mathrm{~ms}$ (patients: $\mathrm{M}=658 \mathrm{~ms}$, $\mathrm{SD}=131 \mathrm{~ms}$; controls: $\mathrm{M}=579 \mathrm{~ms}, \mathrm{SD}=127 \mathrm{~ms}$ ). Thus, for all individuals, the speed of the metronome was varied between Inter-Response-Intervals (IRI) of 300 and 110oms and participants were invited to produce a series of 60 touchdowns at each tempo. The explicit instruction was as follows: "Point each target, one after the other, following the speed set by the metronome. You must be as accurate as possible in space but when in difficulty, make sure you are always in time with the metronome." Resting periods were given periodically to limit arm and cognitive fatigue.

Patients with schizophrenia and controls performed the task without apparent difficulty for 8 out of the 9 tempi. There were no significant differences for IRI errors between controls and patients at all tempi except for the fastest (300ms). For both groups, the fastest tempo was difficult to perform and was characterized by significant increases in IRI errors. These results are presented in Figure 6.5a. At the faster tempi of 400 and $300 \mathrm{~ms}$, many participants reported needing to be highly concentrated and to accept to "let go" in order to maintain rhythmic synchrony. Patients were characterized by larger spatial errors (area) throughout. However, longer contact times were revealed at slower tempi only (from ITI $=700$ to $1100 \mathrm{~ms}$ ). Contrary to what would have been expected in patients characterized by general slowness, our patients with schizophrenia performed as the controls for the faster tempi (from ITI $=300$ to 6ooms - see Figure 6.5b).

It is possible that two different temporal processes are used for faster tempi (from ITI $=300$ to $700 \mathrm{~ms})$, on the one hand, and slower tempi (from ITI $=700$ to 
110oms), on the other hand. Following this idea, patients with schizophrenia would be impaired because of a specific difficulty in the timing of those slow tempi that required (1) the inhibition of spontaneous response and (2) a more cognitive control of timing for long intervals. A first indication of this would be the indication of contrasting cognitive strategies in these two different tempi intervals. The analysis of endpoint distributions (orientation bias) in the healthy controls confirmed that the spatial ellipses were significantly more oriented at faster tempi (ITI < 6ooms), in reference to the straight line connecting successive targets, than at the slower tempi (i.e., smaller errors to the predicted model). As proposed by Desmurget (1998), we suggest that these results reveal the use of an internal representation of the grouped targets, which was used to plan and execute fast sequential actions without the needs to focus attention on each individual target; the temporal processes used here would be emergent and characteristic of the time needed for motor loops to initiate each step of the sequence. At slower tempi however, participants individuated each target more and as such, there was an absence of orientation of the distribution of the endpoint errors (see Figure 6.5c). The change from one type of temporal process to the other appears to be around the critical spontaneous tempo (i.e., 6ooms). Interestingly, our patients with schizophrenia revealed similar results except around the critical spontaneous tempo and seemed to switch timing processes faster than the controls, with more oriented endpoint distributions than that observed in the controls, at the intervals of 600 and 70oms. It is possible that switching strategies occurred faster in the patients from predictive timing to emergent timing in order to engage faster in the emergent timing strategy that would be preserved. This may be also due to perturbed inhibitory functions that are frequently reported in schizophrenia.

Overall, our results suggest the existence of two fundamentally different timing processes with a change in the interplay around the critical moment of preferred tempo. In schizophrenia, the brain mechanism for emergent timing would be preserved. Indeed, patients revealed normalized performances both in time and space compared to adult controls, even if at these fast tempi all participants found the task difficult to achieve. The brain mechanism for predictive timing however seemed to be perturbed in our patients. With a difficulty to inhibit direct response, timing errors were significantly greater than that observed in controls; patients also were characterised with higher performance variability. This pattern of results strongly resembles those reported in the first two sections of this chapter; they also echo recent results published by our co-workers reporting a specific problem in predictive timing using a phase shift detection paradigm (Turgeon et al., 2012). 

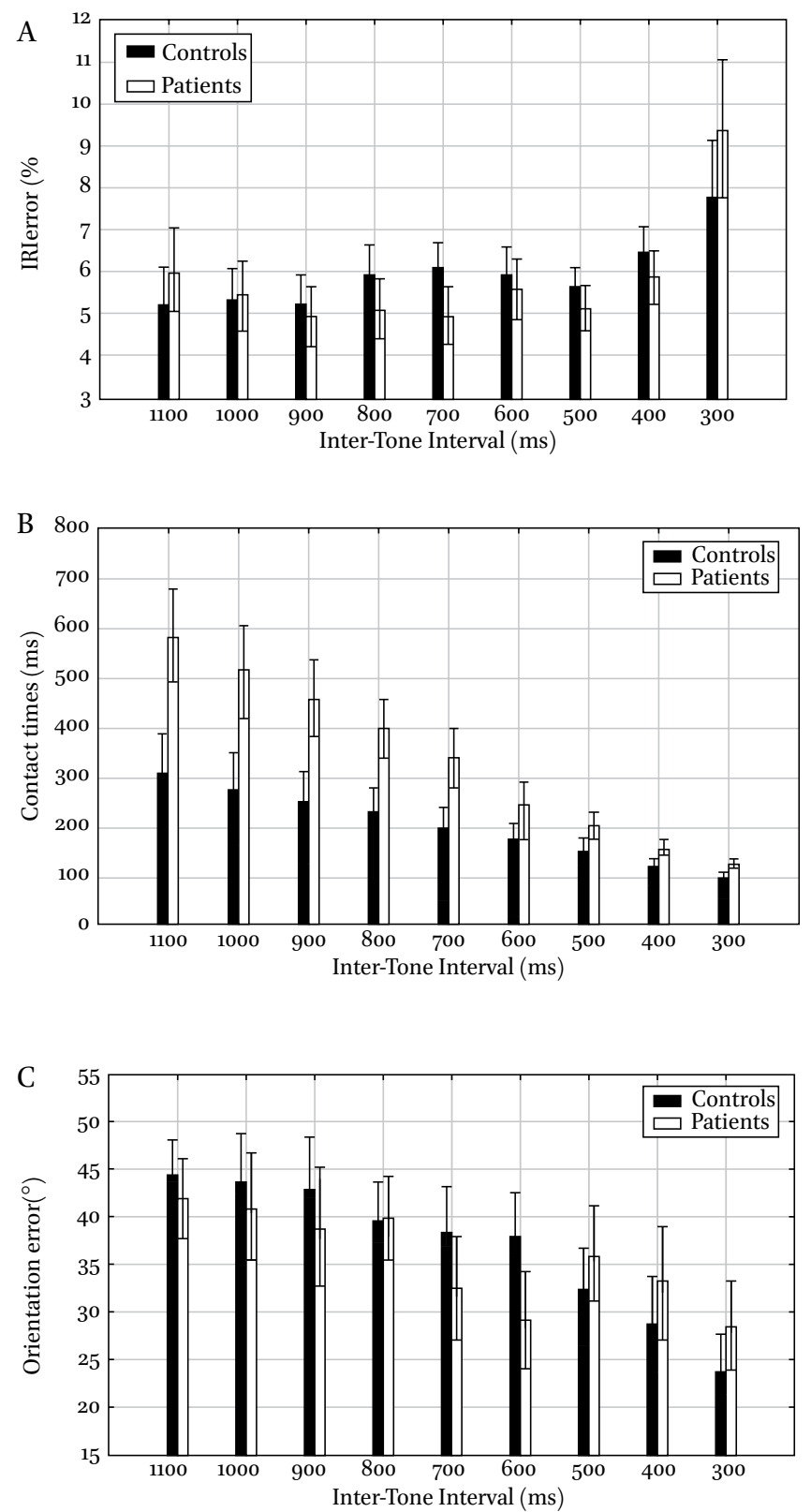

FIGURE 6.5 Results obtained in the Spatio-tapping task in controls (grey) and patients with schizophrenia (black). The top figure (6.5a) illustrates the inter-tap interval error (ITI in ms) compared to the target inter response interval (IRI in ms) that was imposed by a regular metronome. The middle figure (6.5b) presents mean results obtained for the Contact times ( $c$ i in ms) measured for the 11 different tempi. Finally, the bottom figure (6.5c) presents the mean orientations of the endpoint error distributions calculated for oriented scatter plots towards the next target. Large values suggest an absence of orientation. 
In conclusion, two fundamentally different timing processes may exist for both perceptual and motor timing. Repp and Steinman (2010) have recently furthermore argued that these two mechanisms co-exist within the same task. They demonstrated that when carried out simultaneously in synchrony, discrete and continuous tasks (finger-tapping vs. circle drawing) could affect each other in some ways but retain their distinctive timing characteristics. This strongly suggests that predictive timing and emergent timing processes can coexist, with one mode dominating the other as a function of task timing properties. Our findings suggest that schizophrenia may affect specifically those processes involved in predictive timing with the behavioural consequence of significantly perturbing those tasks that require predictive timing, i.e., estimate interval durations in an explicit way; because of the coexisting properties, patients with schizophrenia would also be mildly perturbed in tasks requiring emergent-timing but especially for performance stability, indicator of the event-based aspect of the task. This hypothesis needs now to be tested using both behavioural and brain imaging techniques.

\section{General Conclusions}

Attempts to understand the underlying pathophysiology of schizophrenia have increasingly emphasized disturbances in the temporal coordination of information processing in the brain (Tononi and Edelman, 2000), leading to sensory and motor timing dysfunctions (Carroll et al., 2008; Elvevåg et al., 2003; Penney et al., 200o). In the present chapter, we reviewed a number of studies that investigated interval timing in patients with schizophrenia. Through the use of objective measures of temporal processing capacities, we have shown that the motor fluency deficits in schizophrenia may be due to a deficit in predictive timing and not to a general slowing or instability of the internal clock. The use of new technologies (e.g., tactile screens) will provide the means to develop new tools that will help gain a neuropsychological description of the abnormalities in the phenomenology of timing in patients suffering from brain dysfunctions.

Because the neural circuits that are required for interval timing and those thought to be compromised in schizophrenia overlap, it has been proposed that a focus on temporal information processing offers a window into understanding the cognitive deficits of schizophrenia and how deficits may contribute to a variety of clinical symptoms (Ward et al., 2012). In our laboratory, we are specifically interested in understating the functional overlap that may exist 
between predictive timing and anticipatory motor control, which seem to be two processes that are necessary for a normalised sense of agency (Jardri et al., 2009) and a stable construct of self as a unity of being (Wilquin and DelevoyeTurrell, 2012).

\section{References}

Ameller, A. et al. 2011. "Production de rythmes dans la schizophrénie : Rôle central de l'attention" L'Encéphale.

Andreasen, N.C., S. Paradiso, and D.S. O'Leary 1998. 'Cognitive Dysmetria' as an Integrative Theory of Schizophrenia: A Dysfunction in Cortical-SubcorticalCerebellar Circuitry?" Schizophrenia Bulletin 24(2): 203-18.

Andreasen, Nancy C. et al. 1999. "Defining the Phenotype of Schizophrenia: Cognitive Dysmetria and Its Neural Mechanisms" Biological Psychiatry 46(7): 908-20.

Aschersleben, Gisa. 2002. "Temporal Control of Movements in Sensorimotor Synchronization" Brain and Cognition 48(1): 66-79.

Augustin. 1942. Les confessions de saint Augustin.

Bellak, L. 1949. "A Multiple-Factor Psychosomatic Theory of Schizophrenia; An Attempt at a Consistent Conceptualization" The Psychiatric Quarterly 23(4): $738-55$.

Billon, M.A., Semjen, J. Cole, and G. Gauthier. 1996. "The Role of Sensory Information in the Production of Periodic Finger-Tapping Sequences" Experimental Brain Research. 110(1): 117-30.

Bindra, D., and H. Waksberg. 1956. "Methods and Terminology in Studies of Time Estimation" Psychological Bulletin 53(2): 155-9.

Brébion, G. et al. 2000. "Depression, Psychomotor Retardation, Negative Symptoms, and Memory in Schizophrenia" Neuropsychiatry, Neuropsychology, and Behavioral Neurology 13(3): 177-83.

Brickenkamp, R. 1962. Testd2. Aufmerksamkeits-Belastungs-Test. Hogrefe Verlag, Göttingen.

Brown, S.M. et al. 2005. "Eyeblink Conditioning Deficits Indicate Timing and Cerebellar Abnormalities in Schizophrenia" Brain and Cognition 58(1): 94-108.

Buonomano, D.V. 200o. "Decoding Temporal Information: A Model Based on ShortTerm Synaptic Plasticity" The Journal of Neuroscience: The Official Journal of the Society for Neuroscience 20(3): 1129-41.

Buonomano, D.V., and M.M. Merzenich. 1995. "Temporal Information Transformed into a Spatial Code by a Neural Network with Realistic Properties" Science $267(5200): 1028-30$. 
Buonomano, Dean V., and Rodrigo Laje. 2010. "Population Clocks: Motor Timing with

Neural Dynamics" Trends in Cognitive Sciences 14(12): 520-7.

Burr, David, Arianna Tozzi, and M. Concetta Morrone. 2007. "Neural Mechanisms for

Timing Visual Events are Spatially Selective in Real-World Coordinates" Nature Neuroscience 10(4): 423-5.

Carroll, Christine A. et al. 2008. "Temporal Processing Dysfunction in Schizophrenia" Brain and Cognition 67(2): 150-61.

Carroll, Christine A. et al. 2009a. "Timing Dysfunctions in Schizophrenia Span from Millisecond to Several-Second Durations" Brain and Cognition 70(2): 181-90.

Carroll, Christine A. et al. 20ogb. "Timing Dysfunctions in Schizophrenia as Measured by a Repetitive Finger Tapping Task". Brain and Cognition 71(3): 345-53.

Clausen, J. 1950. "An Evaluation of Experimental Methods of Time Judgment" Journal of Experimental Psychology 40(6): 756-61.

Cohen, J.D., and D. Servan-Schreiber. 1992. "Context, Cortex, and Dopamine: A Connectionist Approach to Behavior and Biology in Schizophrenia" Psychological Review 99(1): 45-77.

Cornblatt, B.A., and J.G. Keilp. 1994. "Impaired Attention, Genetics, and the Pathophysiology of Schizophrenia" Schizophrenia Bulletin 20(1): 31-46.

Creelman, C. Douglas. 1962. "Human Discrimination of Auditory Duration" The Journal of the Acoustical Society of America 34(5): 582-93.

Cumming, W.J. 1988. "The Neurobiology of the Body Schema" The British Journal of Psychiatry 2: 7-11.

Davalos, Deana B., Michael A. Kisley, and Randal G. Ross. 2003. "Effects of Interval Duration on Temporal Processing in Schizophrenia" Brain and Cognition 52(3): 295-301.

Delevoye-Turrell, Yvonne, Anne Giersch, and Jean-Marie Danion. 2003. "Abnormal Sequencing of Motor Actions in Patients with Schizophrenia: Evidence from Grip Force Adjustments During Object Manipulation" The American Journal of Psychiatry 16o(1): 134-41.

Delevoye-Turrell, Yvonne Nathalie, Pierre Thomas, and Anne Giersch. 2006. "Attention for Movement Production: Abnormal Profiles in Schizophrenia" Schizophrenia Research 84(2-3): 430-2.

Delevoye-Turrell, Yvonne et al. 2007. "Motor Fluency Deficits in the Sequencing of Actions in Schizophrenia" Journal of Abnormal Psychology 116(1): 56-64.

Delevoye-Turrell, Yvonne, Hélène Wilquin, and Anne Giersch. 2012. "A Ticking Clock for the Production of Sequential Actions: Where Does the Problem Lie in Schizophrenia?" Schizophrenia Research 135(1-3): 51-4.

Delignières, Didier, Loïc Lemoine, and Kjerstin Torre. 2004. “Time Intervals Production in Tapping and Oscillatory Motion" Human Movement Science 23(2): 87-103.

Densen, M.E. 1977. "Time Perception and Schizophrenia” Perceptual and Motor Skills 44(2): $436-8$. 
Desmurget, M. et al. 1998. "From Eye to Hand: Planning Goal-Directed Movements" Neuroscience and Biobehavioral Reviews 22(6): 761-88.

Elvevåg, B. et al. 2003. "Duration Judgements in Patients with Schizophrenia" Psychological Medicine 33(7): 1249-61.

Elvevåg, B. et al. 2004. "Identification of Tone Duration, Line Length, and Letter Position: An Experimental Approach to Timing and Working Memory Deficits in Schizophrenia" Journal of Abnormal Psychology 113(4): 509-21.

Fisher, Seymour, Stephen G. Bryant, and Ronica M. Kluge. 1986. "New Approaches to Postmarketing Surveillance" Psychopharmacology 9o(3).

Fleming, K. et al. 1997. "Visuospatial Working Memory in Patients with Schizophrenia" Biological Psychiatry 41(1): 43-9.

Fortin, C., and N. Massé. 1999. "Order Information in Short-Term Memory and Time Estimation" Memory and Cognition 27(1): 54-62.

Fortin, C. et al. 1993. "Time Estimation and Concurrent Nontemporal Processing: Specific Interference from Short-Term-Memory Demands" Perception and Psychophysics 53(5): 536-48.

Freedman, B.J. 1974. "The Subjective Experience of Perceptual and Cognitive Disturbances in Schizophrenia. A Review of Autobiographical Accounts" Archives of General Psychiatry 30(3): 333-340.

Gibbon, J., and Church, R.M. 1984. "Sources of Variance in an Information Processing Theory of Timing. " In H.L. Roitblat, T.G. Bever, and H.S. Terrace (Eds), Animal Cognition 465-88. Hillsdale, NJ: Erlbaum.

Giersch, Anne et al. 2009. "Extended Visual Simultaneity Thresholds in Patients with Schizophrenia" Schizophrenia Bulletin 35(4): 816-25.

Goldstone, S., and W.T. Lhamon. 1956. "The Time Sense: Estimation of One Second Durations by Schizophrenic Patients" A.M A. Archives of Neurology and Psychiatry 76(6): 625-9.

Gordon, J., M.F. Ghilardi, and C. Ghez. 1994. "Accuracy of Planar Reaching Movements. I. Independence of Direction and Extent Variability" Experimental Brain Research 99(1): 97-111.

Grondin, S. 2010. "Timing and Time Perception: A Review of Recent Behavioral and Neuroscience Findings and Theoretical Directions" Attention, Perception, and Psychophysics 72(3): 561-82.

Gurfinkel, V.S. et al. 1989. "[Changes in the Direction of Vestibulomotor Responses in the Process of Adaptation to Prolonged Static Head Turning in Man.]" Neiřfiziologiia Neurophysiology 21(2): 210-7.

Harrow, Martin, and Thomas H. Jobe. 2007. "Factors Involved in Outcome and Recovery in Schizophrenia Patients Not on Antipsychotic Medications: A 15-Year Multifollow-Up Study" The Journal of Nervous and Mental Disease 195(5): 406-14. 
Howes, Oliver D., and Shitij Kapur. 2009. “The Dopamine Hypothesis of Schizophrenia: Version III - The Final Common Pathway" Schizophrenia Bulletin 35(3): 549-62.

Ivry, R. 1997. "Cerebellar Timing Systems" International Review of Neurobiology 41: $555^{-73}$.

Ivry, R.B., and R.E. Hazeltine. 1995. "Perception and Production of Temporal Intervals Across a Range of Durations: Evidence for a Common Timing Mechanism" Journalof Experimental Psychology. Human Perception andPerformance $21(1): 3^{-18}$.

Ivry, R.B., S.W. Keele, and H.C. Diener. 1988. "Dissociation of the Lateral and Medial Cerebellum in Movement Timing and Movement Execution" Experimental Brain Research 73(1): 167-80.

Jardri, Renaud et al. 2009. "Clinical Practice of rTMS Reveals a Functional Dissociation between Agency and Hallucinations in Schizophrenia” Neuropsychologia 47(1): $132-8$.

Johnson, J.E., and T.P. Petzel. 1971. "Temporal Orientation and Time Estimation in Chronic Schizophrenics" Journal of Clinical Psychology 27(2): 194-6.

Kant, E. 1845. Critique de la raison pure. Ladrange.

Karson, C.N. 1983. "Spontaneous Eye-Blink Rates and Dopaminergic Systems" Brain: A Journal of Neurology 106(3): 643-53.

Knoblich, Günther, Frank Stottmeister, and Tilo Kircher. 2004. "Self-Monitoring in Patients with Schizophrenia" Psychological Medicine 34(8): 1561-9.

Lalanne, Laurence, Mitsouko van Assche, and Anne Giersch. 2010. "When Predictive Mechanisms Go Wrong: Disordered Visual Synchrony Thresholds in Schizophrenia" Schizophrenia Bulletin.

Lee, Kwang-Hyuk et al. 2009. "Time Perception and Its Neuropsychological Correlates in Patients with Schizophrenia and in Healthy Volunteers" Psychiatry Research 166(2-3): 174-83.

Lewis, Aubrey. 1932. "The Experience of Time in Mental Disorder" Proceedings of the Royal Society of Medicine 25(5): 611-20.

Maass, Wolfgang, Thomas Natschläger, and Henry Markram. 2002. "Real-Time Computing without Stable States: A New Framework for Neural Computation Based on Perturbations" Neural Computation 14(11): 2531-60.

Macefield, V.G., and R.S. Johansson. 1994. "Electrical Signs of Cortical Involvement in the Automatic Control of Grip Force" Neuroreport 5(17): 2229-32.

McAuley, J. Devin et al. 2006. "The Time of Our Lives: Life Span Development of Timing and Event Tracking" Journal of Experimental PsychologyGeneral. 135(3): 348-67.

Meck, W H. 1996. "Neuropharmacology of Timing and Time Perception" Cognitive Brain Research 3(3-4): 227-42. 
Minkowski, Eugène. 1933. Le temps vécu: études phénoménologiques and psychopathologiques. Collection de l'évolution psychiatrique.

Morrens, Manuel, Wouter Hulstijn, and Bernard Sabbe. 2007. "Psychomotor Slowing in Schizophrenia" Schizophrenia Bulletin 33(4): 1038-53.

Newton, Isaac. 1987. Philosophiae naturalis principia mathematica.

Nuechterlein, K.H. 1977. "Reaction Time and Attention in Schizophrenia: A Critical Evaluation of the Data and Theories" Schizophrenia Bulletin 3(3): 373-428.

Nuechterlein, K.H, and M.E. Dawson. 1984. "Information Processing and Attentional Functioning in the Developmental Course of Schizophrenic Disorders" Schizophrenia Bulletin 10(2): 160-203.

Penney, T.B., J. Gibbon, and W.H. Meck. 2000. "Differential Effects of Auditory and Visual Signals on Clock Speed and Temporal Memory" Journal of Experimental Psychology. Human Perception and Performance 26(6): 1770-87.

Pressing, J. 1998. "Referential Behaviour Theory: A Framework for Multiple Perspectives on Motor Control. Motor Behavior and Human Skill: A Multidisciplinary Approach" 357-84.

Pressing, Jeff. 1999. "The Referential Dynamics of Cognition and Action" Psychological Review 106(4): 714-47.

Priebe, Stefan, and Frank Röhricht. 2001. "Specific Body Image Pathology in Acute Schizophrenia" Psychiatry Research 101(3): 289-301.

Rammsayer, T. 1990. "Temporal Discrimination in Schizophrenic and Affective Disorders: Evidence for a Dopamine-Dependent Internal Clock" The International Journal of Neuroscience 53(2-4): 111-20.

Rammsayer, T.H. 1999. "Neuropharmacological Evidence for Different Timing Mechanisms in Humans" The Quarterly Journal of Experimental Psychology. B, Comparative and Physiological Psychology 52(3): 273-86.

Repp, Bruno H. 2005. "Sensorimotor Synchronization: A Review of the Tapping Literature" Psychonomic Bulletin and Review 12(6): 969-92.

Repp, Bruno H., and Susan R. Steinman. 2010. "Simultaneous Event-Based and Emergent Timing: Synchronization, Continuation, and Phase Correction" Journal of Motor Behavior 42(2): 111-26.

Robertson, Shannon D. et al. 1999. "Correlations for Timing Consistency among Tapping and Drawing Tasks: Evidence against a Single Timing Process for Motor Control ". Journal of Experimental Psychology: Human Perception and Performance 25(5): 1316-30.

Stevens, L.T. 1986. "On the Time-Sense" Mind 43: 393.

Stone, M. et al. 1998. "Working and Strategic Memory Deficits in Schizophrenia" Neuropsychology 12(2): 278-88.

Tononi, G., and G.M. Edelman. 200o. "Schizophrenia and the Mechanisms of Conscious Integration" Brain Research. Brain Research Reviews 31(2-3): 391-400. 
Treisman, M.1963. “Temporal Discrimination and the Indifference Interval. Implications for a Model of the 'Internal Clock"' Psychological Monographs 77(13): 1-31.

Tsuang, M.T., W.S. Stone, and S.V. Faraone. 2001. "Genes, Environment and Schizophrenia” The British journal of psychiatry. Supplement 40: S18-24.

Turgeon, Martine et al. 2012. "Impaired Predictive Timing with Spared Time Interval Production in Individual with Schizophrenia" Psychiatry Research 197(1-2): $13-8$.

Tysk, L. 1983a. "Estimation of Time and the Sub Classification of Schizophrenic Disorders" Perceptual and Motor Skills 57(3): 911-8.

Tysk, L. 1983b. "Time Estimation by Healthy Participants and Schizophrenic Patients: A Methodological Study" Perceptual and Motor Skills 56(3): 983-8.

Tysk, L. 199o. "Estimation of Time by Patients with Positive and Negative Schizophrenia" Perceptual and Motor Skills 71(3): 826.

Vanneste, S., V. Pouthas, and J.H. Wearden. 2001. "Temporal Control of Rhythmic Performance: A Comparison between Young and Old Adults" Experimental Aging Research 27(1): 83-102.

Vindras, P, and P. Viviani. 1998. "Frames of Reference and Control Parameters in Visuomanual Pointing" Journal of Experimental Psychology. Human Perception and Performance 24(2): 569-91.

Volz, H.P. et al. 2001. "Time Estimation in Schizophrenia: An fMRI Study at Adjusted Levels of Difficulty" Neuroreport 12(2): 313-6.

Wahl, O.F., and D. Sieg. 1980. "Time Estimation among Schizophrenics" Perceptual and Motor Skills 50(2): 535-41.

Ward, Ryan D. et al. 2012. "Timing as a Window on Cognition in Schizophrenia" Neuropharmacology 62(3): 1175-81.

Wilquin, Hélène, and Yvonne Delevoye-Turrell. 2012. "Motor Agency: A New and Highly Sensitive Measure to Reveal Agency Disturbances in Early Psychosis” PloS One 7(2): e30449.

Wilquin, H. et al. 2010. "Production de rythmes dans la schizophrénie: Un déficit de l'alternance de temps" L'Année psychologique 110(4): 517-39.

Wing, A., and Kristofferson, A. 1973. "The Timing of Interresponse Intervals" Attention, Perception, and Psychophysics 13(3): 455-6o.

Zahn, T.P. et al. 1998. "Manual and Saccadic Reaction time with Constant and Variable Preparatory Intervals in Schizophrenia" Journal of Abnormal Psychology 107(2): 328-37.

Zakay, Dan, and Richard A. Block. 1996. "The Role of Attention in Time Estimation Processes" In Time, Internal Clocks and Movement, 115:143-64. North-Holland.

Zelaznik, Howard N., Rebecca M.C. Spencer, and Richard B Ivry. 2002. "Dissociation of Explicit and Implicit Timing in Repetitive Tapping and Drawing Movements" 
Journal of Experimental Psychology. Human Perception and Performance 28(3): $575^{-88 .}$

Zelaznik, H., Spencer, R., and Doffin, J. 200o. “Temporal Precision in Tapping and Circle Drawing Movements at Preferred Rates is Not Correlated: Further Evidence against Timing as a General-Purpose Ability" Journal of motor behavior 32(2): 193-9.

Zelaznik, Howard N. et al. 2005. "Timing Variability in Circle Drawing and Tapping: Probing the Relationship between Event and Emergent Timing" Journal of Motor Behavior 37(5): 395-403. 